\title{
Advanced battery management systems using fast electrochemical modelling
}

\author{
A Bizeray*, SR Duncan ${ }^{\dagger}$, DA Howey* \\ *University of Oxford - Energy and Power Group, UK, adrien.bizeray@eng.ox.ac.uk, \\ ${ }^{\dagger}$ University of Oxford - Control Group, $U K$
}

Keywords: Lithium-ion, battery modelling, battery management systems, spectral orthogonal collocation

\begin{abstract}
This paper presents a novel approach using a spectral orthogonal collocation method to solve the well-established 'Newman' model for lithium-ion batteries. The resulting system of equations is much lower order and faster to solve than the equation system obtained using the more common finite difference method, but retains the accuracy of the model, with simulation results in good agreement up to high C-rates (40C) compared to the finite difference approach. This approach enables real-time solution of the model to become possible leading to efficient and accurate on-line estimation of physically meaningful states in an advanced battery management system.
\end{abstract}

\section{Introduction}

Due to their high power and energy density, lithium-ion batteries have a promising future in energy storage for electric vehicles (EVs) and hybrid electric vehicles (HEVs). Lithiumion batteries are relatively expensive and must operate within narrow voltage, current and temperature safety limits to avoid premature aging or failure. Safety and degradation issues become particularly acute at high C-rate charging and discharging, which is commonly encountered during high performance $\mathrm{HEV}$ operation or fast charging. Battery management systems (BMSs) are designed to ensure that the battery pack gives best performance and operates within safety limits, by monitoring states of battery cells such as state-of-charge (SOC), pulse power capability (PPC), state-ofhealth $(\mathrm{SOH})$, etc. However, these cannot be measured directly but are calculated using a model, from external measurements of voltage, current and temperature. Current BMSs use crude empirical models such as experimentallyparameterized equivalent circuit models. These have no physical meaning and become unusable in extreme operating conditions (e.g. high and low temperatures, high C-rate charge/discharge). Unlike empirical models, fundamental models which relate transport of ions and reaction kinetics to voltage and current are able to accurately estimate essential parameters and safety limits over a wide operating range. These have been widely used for battery design and optimization but their implementation in embedded BMSs is challenging due to their complexity and high computational requirements.
The so-called Newman model developed by Doyle et al. [1] is probably the most widely used lithium-ion battery fundamental model. This is a one-dimensional physics-based electrochemical model that consists of a set of coupled governing equations describing thermodynamics, transport phenomena and kinetics of a cell. Porous electrode theory models the electrodes as a continuum of superimposed phases with averaged properties. To account for solid diffusion, a solid spherical particle is assumed at each discrete node of the model and the diffusion equation is solved on a pseudosecond dimension along the particles' radii. Therefore, the Newman model is sometimes referred to as the pseudo-two dimensional (P2D) model. The P2D model is able to capture lithium-ion dynamics accurately by modelling diffusion and kinetics, and is therefore relevant for highly dynamic loads and is an excellent starting point for the next generation of BMSs [2].

Several simplification techniques have been explored in the literature to reduce the computational burden of the P2D model. Firstly, solving the diffusion equation in solid particles at each node of the model drastically increases the model complexity and extensive literature has been published on locally simplifying this micro-scale model. Approaches including assuming a parabolic concentration profile [3], using a truncated series solution [4], and frequency domain residue grouping [5] have been used. However, these suffer limitations such as only being valid at low C-rates $(<10 \mathrm{C})$ or requiring calculation of a complementary error function $e r f c$. Alternatively, each electrode may be assumed to be a single particle, with constant electrolyte concentration [6]. However, this is only valid at low C-rates $(<5 \mathrm{C})$. Secondly, several model order reduction techniques have been applied for globally simplifying the P2D model: Smith et al. [7] developed a reduced order state variable model based on linearized transfer functions and Cai and White [8] proposed a projection technique called Proper Orthogonal Decomposition. However, the physical meaning of the model is lost in these model order reduction processes, which means that it has to be parameterized again if the battery behaviour changes due to aging for example.

All of these approaches lose information and physical meaning compared to the full P2D model. We therefore seek an approach that maintains the fidelity of the P2D model but is computationally fast. The P2D model consists of a set of non-linear time-varying PDEs with algebraic constraints. The Finite Difference Method (FDM) is often used to discretize such PDEs in space. Unlike the FDM, spectral methods use a global approximation of the derivatives by assuming that the 
solution of the PDE can be expressed as a sum of orthogonal basis functions. The main advantage of spectral methods is that the same accuracy can be obtained with far fewer discrete nodes than are required for the FDM (reduced by a factor of $10 \sim 100)$ provided that the solution is smooth enough. Dao et al. [9] used the Galerkin spectral method with sinusoidal basis functions to solve the electrolyte diffusion equation. However, restrictive assumptions were made including a parabolic solid-phase concentration profile [3] and uniform reaction rate within electrodes, which limit the validity to low C-rates. Northrop et al. [10] used a pseudo-spectral method, also known as a collocation spectral method, to solve all the equations of the model with Jacobi polynomial basis functions using DASSL and Maple solvers. They confirmed that the computation time can be reduced by a factor of 10 to 100 compared to the FDM with a similar accuracy. This computational cost reduction allowed them to run the P2D model for a battery stack comprising 8 cells in a reasonable computation time. Cai and White [11] developed a similar P2D model but using collocation spectral methods on finite elements. They showed that solving the P2D model using this method is 30 times faster than solving it using FDM with COMSOL Multiphysics ${ }^{\circledR}$.

In this paper, the implementation of the isothermal P2D model using spectral orthogonal collocation will be discussed. Our approach is novel because it is unified, i.e. all the equations are solved using the same technique with no simplifying assumptions. The spectral differentiation matrix approach presented by Trefethen [12] was used with the Matlab differentiation suite developed by Weideman and Reddy [13]. The model therefore reduces to a set of ordinary differential equations and algebraic constraints that can be solved by a Matlab ODE solver, guaranteeing a fast solution and easy implementation in an embedded system.

\section{Mathematical model of the insertion battery}

A lithium-ion battery cell consists of two porous electrodes that can store lithium and a separator that allows the passage of lithium ions but not electrons. From a modelling perspective, the battery cell can be divided into three domains, anode, separator and cathode, respectively denoted by the subscripts 1,2 and 3 in the present paper. In each of these domains, two phases can be encountered, the solidphase and the electrolyte phase denoted by the subscripts $s$ and $e$ respectively. However, each domain is treated as a continuum with averaged properties calculated according the volume fraction of each phase.

$$
\mathrm{Li}^{+}+e^{-}+\theta_{s} \leftrightharpoons \operatorname{Li}_{s}
$$

Lithium-ion batteries involve an insertion/de-insertion process of lithium into the active material of the electrodes described by Equation (1), where $\theta_{s}$ represents an insertion site in the electrode. During discharge (Figure 1), lithium is de-inserted from the anode and migrates to the cathode as ions in the electrolyte driven by concentration and electric potential gradients. When lithium ions reach the cathode, they are inserted in the cathode material. Simultaneously, electrons transfer from the anode to the cathode through the external circuit to maintain the material electroneutrality, yielding an electric current. The process is reversed during charging.

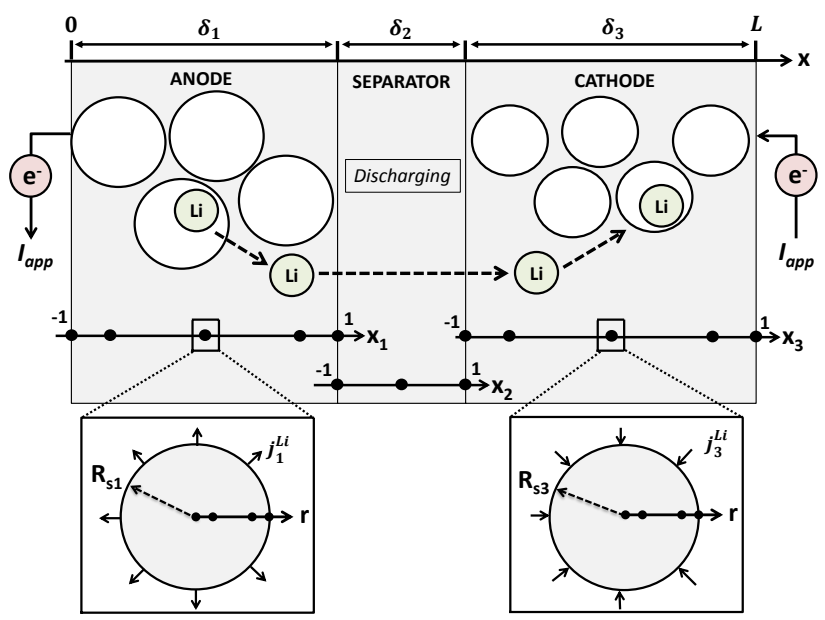

Figure 1: Schematics of the battery geometry with the Chebyshev collocation points.

\subsection{Governing equations}

The P2D model describes the spatial distribution and temporal evolution of lithium concentration in the electrolyte and solid phase $c_{e}(x, t)$ and $c_{s}(x, t)$ as well as the electrolyte and solid phase electric potential $\phi_{e}(x, t)$ and $\phi_{s}(x, t)$ respectively. The amount of lithium exchanged between the solid phase and the electrolyte is given by the volumetric rate of reaction $j^{L i}(x, t)$ estimated by the Butler-Volmer Equation (2) as a function of the overpotential $\eta(x, t)$.

$$
j^{L i}=a_{s} i_{0}\left\{\exp \left[\frac{\alpha_{a} \mathcal{F}}{R T} \eta\right]-\exp \left[\frac{-\alpha_{c} \mathcal{F}}{R T} \eta\right]\right\}
$$

The exchange current density $i_{0}(x, t)$ in Equation (2) depends on the electrode reaction rate constant $k$ and the local solidphase and electrolyte concentrations according to:

$$
i_{0}=k \mathcal{F}\left(c_{s}^{\max }-c_{s}^{\text {surf }}\right)^{\alpha_{a}}\left(c_{s}^{\text {surf }}\right)^{\alpha_{c}}\left(c_{e}\right)^{\alpha_{a}}
$$

The overpotential $\eta(x, t)$ is defined in Equation (4) where the open-circuit potential $U^{o c p}$ is an experimentally-fitted function of the stoichiometry $\theta$ for each electrode.

$$
\eta=\phi_{s}-\phi_{e}-U^{o c p}(\theta)
$$

At each node $\left\{x_{k}: k=0,1, \ldots, N\right\}$, the solid-phase concentration within a spherical particle of electrode active material is governed by Equation (5), which is the diffusion equation in spherical coordinates.

$$
\begin{gathered}
\frac{\partial c_{s}}{\partial t}=\frac{D_{s}}{r^{2}} \frac{\partial}{\partial r}\left(r^{2} \frac{\partial c_{s}}{\partial r}\right) \\
\left.\frac{\partial c_{s}}{\partial r}\right|_{r=0}=0 \quad \text { and }\left.\quad D_{s} \frac{\partial c_{s}}{\partial r}\right|_{r=R_{s}}=\frac{-j^{L i}}{a_{s} \mathcal{F}}
\end{gathered}
$$

Equation (6) gives the boundary conditions at the centre and at the surface of the particles, where $a_{s}=3 \epsilon_{s} / R_{s}$ is the specific 
interfacial area of the electrode (surface area per unit volume of electrode).

Conservation of lithium in the electrolyte leads to Equation (7) governing $c_{e}(x, t)$, where $D_{e}^{\text {eff }}=\epsilon_{e}^{p} D_{e}$ is the effective diffusion coefficient accounting for the material porosity.

$$
\epsilon_{e} \frac{\partial c_{e}}{\partial t}=D_{e}^{e f f} \frac{\partial^{2} c_{e}}{\partial x^{2}}+\frac{1-t_{+}^{0}}{\mathcal{F}} j^{L i}
$$

This is subject to a zero-flux boundary condition, which expresses the fact that no lithium ions can go through the current collectors:

$$
\left.\frac{\partial c_{e}}{\partial x}\right|_{x=0}=\left.\frac{\partial c_{e}}{\partial x}\right|_{x=L}=0
$$

Conservation of charge in the solid-phase material yields to the Ohm's law given by Equation (9) that governs the solidphase electric potential, where $\sigma^{e f f}=\epsilon_{s} \sigma$ is the effective solidphase conductivity.

$$
\sigma^{e f f} \frac{\partial^{2} \phi_{s}}{\partial x^{2}}-j^{L i}=0
$$

At the interface with the current collectors, the flux boundary condition is equal to the applied current density given by Equation (10). At the electrode/electrolyte interface, the flux boundary condition expressed by Equation (11) is equal to zero since all the current is carried by ions.

$$
\begin{gathered}
\left.\sigma_{1}^{e f f} \frac{\partial \phi_{s}}{\partial x}\right|_{x=0}=\left.\sigma_{3}^{e f f} \frac{\partial \phi_{s}}{\partial x}\right|_{x=L}=-\frac{I_{a p p}}{A} \\
\left.\frac{\partial \phi_{s}}{\partial x}\right|_{x=\delta_{1}}=\left.\frac{\partial \phi_{s}}{\partial x}\right|_{x=\delta_{1}+\delta_{2}}=0
\end{gathered}
$$

The conservation of charge in the electrolyte is described by Ohm's law, corrected for the gradient of concentration in electrolyte:

$$
\frac{\partial^{2} \phi_{e}}{\partial x^{2}}+\frac{2 R T}{\mathcal{F}}\left(t_{+}^{0}-1\right) \frac{\partial^{2} \ln c_{e}}{\partial x^{2}}+\frac{j^{L i}}{\kappa^{e f f}}=0
$$

The flux boundary conditions at the interfaces with the current collectors are equal to zero since all the current is carried by electrons in the solid-phase, so that:

$$
\left.\frac{\partial \phi_{e}}{\partial x}\right|_{x=0}=\left.\frac{\partial \phi_{e}}{\partial x}\right|_{x=L}=0
$$

The battery terminal voltage is equal to the difference between the solid-phase potential at the cathode current collector and at the anode current collector.

$$
V(t)=\phi_{s}(L, t)-\phi_{s}(0, t)
$$

The P2D model consists of a set of two partial differential equations (PDEs) (Equations (5) and (7)), two ordinary differential equations (ODEs) in space (Equations (9) and (12) ) and one coupling algebraic equation (Equation (2)). This system of equations is usually solved using numerical methods such as a finite difference method (FDM) but in this paper an efficient and faster numerical method called spectral orthogonal collocation is used.

\subsection{Spectral orthogonal collocation method}

Spectral methods are numerical methods for solving ODEs and PDEs that solve the set of equations at discrete nodes. The spectral orthogonal collocation technique is very similar to the FDM since it approximates derivatives of the unknown solution by a polynomial function. The FDM involves local piecewise approximation of the function and its derivatives obtained by Taylor series expansions. This leads to an expression of the solution's derivatives at a node in terms of the value of the solution at the neighbouring nodes. The spectral orthogonal collocation however involves a global approximation of the function and its derivatives as a finite sum of known orthogonal polynomial functions. This yields to an expression of the unknown solution and its derivatives at a node in terms of the value of the solution at all the other nodes of the domain. Provided that the solution of the ODE/PDE is smooth, spectral methods perform with better accuracy and faster speed than the FDM. Therefore, similar accuracy can be obtained with only a few nodes compared to the FDM, which drastically reduces the number of equations to be solved by a factor of 10-100.

\section{Solution interpolation}

The orthogonal collocation spectral method approximates the unknown solution $f$ of an ODE by a polynomial function $p_{N-1}$ of degree $N-1$, which is defined as a finite sum of known polynomial functions $\psi_{j}$ as shown in Equation (15).

$$
f(x) \approx p_{N-1}(x)=\sum_{j=1}^{N} \psi_{j}(x) f\left(x_{j}\right)
$$

A set of orthogonal polynomial basis functions $\left\{\psi_{k}\right\}$ is chosen. In the present work this basis uses Chebyshev polynomials of the first kind $T_{k}$ defined in Equation (16).

$$
T_{k}(\cos \theta)=\cos (k \theta), \quad k=1, \ldots, N
$$

The basis of orthogonal functions $\left\{\psi_{k}\right\}$ is defined in Equation (17) so that the approximating polynomial is interpolating the solution by enforcing the relation $p_{N-1}\left(x_{j}\right)=f\left(x_{j}\right)$, i.e. the value of the approximating polynomial is equal to the value of the solution at the $N-1$ collocation points $x_{j}$.

$$
\begin{gathered}
\psi_{j}(x)=\frac{(-1)^{j}}{c_{j}} \frac{1-x^{2}}{(N-1)^{2}} \frac{T_{N-1}^{\prime}(x)}{x-x_{j}} \\
c_{1}=c_{N}=2 \quad \text { and } \quad c_{2}=\cdots=c_{N-1}=1
\end{gathered}
$$

Once the solution is expressed in terms of its interpolant, deriving the expression of the solutions' derivatives is straightforward and only consists in differentiating the known interpolant so that for the $l^{\text {th }}$ derivative of the solution:

$$
f^{(l)}\left(x_{k}\right) \approx \sum_{j=1}^{N} \frac{d^{l}}{d x^{l}}\left[\psi_{j}(x)\right]_{x=x_{k}} f\left(x_{j}\right), \quad k=1, \ldots, N
$$




\section{Differentiation matrix}

The differentiation operation described in Equation (18) is linear and can therefore be replaced by a discrete differentiation matrix operator. Consequently, the differentiation of the solution $f$ reduces to a matrix-vector multiplication shown in Equation (19), where $v$ (resp. $v^{(l)}$ ) denotes the vector of the solution values (resp. $l^{\text {th }}$ derivative values) at the collocation points $x_{k}$ and the differentiation matrix $D_{N}^{(l)}$ is a square matrix of size $(N-1) \times(N-1)$.

$$
\boldsymbol{v}^{(l)}=D_{N}^{(l)} \boldsymbol{v}
$$

Although the differentiation matrix $D_{N}{ }^{(l)}$ is a full matrix, its size is much smaller compared to the large sparse differentiation matrix computed in the FDM, because only a few collocation points are required for the same accuracy of results. The Matlab differentiation matrix Suite [13] has been used to compute the differentiation matrices. We refer the reader to the literature for more details on the differentiation matrix computation $[12,13]$.

\subsection{P2D model reformulation}

\section{Domain splitting}

The orthogonal collocation method has been used to solve the P2D model in Matlab. However, the use of the collocation method requires some reformulations of the model. Spectral methods are efficient and fast compared to usual numerical methods provided that the solutions of the PDEs are smooth. Therefore, the battery cell has been split into three domains corresponding to the anode, separator and cathode to ensure the smoothness of solutions, i.e. each equation of the P2D model has been solved in each domain separately. The interfaces between electrode and separator introduce discontinuities in the solutions of the PDEs that could significantly reduce the spectral accuracy. For instance, the reaction rate $j^{L i}$ is equal to zero in the separator since no insertion or deinsertion process occurs, but is non-zero at the electrode/separator interface. Solving the equation independently on the three domains requires the introduction of new boundary conditions at the electrode/separator interfaces. Considering the electrolyte concentration $c_{e}$ for example, these new boundary conditions express the continuity of the solution given by Equation (20) and the continuity of the flux given by Equation (21) at the interfaces between the anode and the separator. Similar approaches for the other equations of the model have been followed, but the reformulated equations are not discussed in the present paper; similar reformulated equations of the P2D model are summarized in [10].

$$
\begin{aligned}
c_{e, 1}\left(\delta_{1}, t\right) & =c_{e, 2}\left(\delta_{1}, t\right) \\
\left.D_{e, 1}^{e f f} \frac{\partial c_{e, 1}}{\partial x}\right|_{x=\delta_{1}} & =\left.D_{e, 2}^{e f f} \frac{\partial c_{e, 2}}{\partial x}\right|_{x=\delta_{1}}
\end{aligned}
$$

\section{Coordinate transformation}

A further required reformulation of the model that is inherent to the use of the orthogonal collocation method is rescaling the domains. The collocation points $\left\{x_{k}\right\}$ used in the orthogonal collocation methods are specific nodes called Chebyshev nodes, which correspond to the points of maxima of the Chebyshev polynomials $T_{n}$ used in the interpolant. This set of nodes is clustered at the boundaries of the domain. Chebyshev nodes are defined on $x_{k} \in[-1,1]$,

$$
x_{k}=\cos \left(\frac{(k-1) \pi}{N-1}\right) \text { with } k=1, \ldots, N
$$

so, the coordinates $x_{i} \epsilon\left[0, \delta_{i}\right]$ of each domain have to be mapped onto $[-1,1]$ using the coordinate transformation formula:

$$
\tilde{x}_{i}=\frac{2}{\delta_{i}}\left(x_{i}-\frac{\delta_{i}}{2}\right) \quad \text { with } \quad i=1,2,3
$$

\subsection{Implementation in Matlab}

The reformulated spectral P2D model has been solved in Matlab using a built in ODE/DAE solver, ensuring fast and efficient simulation. The spatial discretization of the P2D model using the orthogonal collocation method reduces the system of equation to a set of ODEs in time for the solidphase concentration and electrolyte concentration and algebraic constraints for the other variables since they are not governed by time dependent differential equations. Therefore, the problem to be solved is a system of differential-algebraic equations (DAEs) that can be rewritten as Equation (24),

$$
\left\{\begin{array}{c}
M \frac{\partial \boldsymbol{y}_{\mathbf{1}}}{\partial t}=g_{1}\left(t, \boldsymbol{y}_{1}, \boldsymbol{y}_{2}\right) \\
0=g_{2}\left(t, \boldsymbol{y}_{1}, \boldsymbol{y}_{2}\right)
\end{array}\right.
$$

Where $\boldsymbol{y}_{\boldsymbol{1}}$ and $\boldsymbol{y}_{2}$ are state vectors containing the values of the states at each collocation node defined in Equation (25). The functions $g_{1}$ and $g_{2}$ are defined using the differentiation matrices previously discussed.

$$
\boldsymbol{y}_{1}=\left[\boldsymbol{c}_{s}, \boldsymbol{c}_{e}\right]^{T} \text { and } \boldsymbol{y}_{2}=\left[\boldsymbol{\phi}_{s}, \boldsymbol{\phi}_{e}\right]^{T}
$$

\section{Simulation results and discussion}

The voltage prediction of our model using spectral orthogonal collocation is compared to the prediction computed by the well-validated Fortran FDM P2D code developed by Newman. The model parameters used for this comparison are summarized in Table 1. The P2D model is solved using 4 collocation points in each electrode, 2 collocation points in the separator and 10 collocation points in each solid particle. This yields to a system of 82 DAEs to be solved. In comparison, the Fortran FDM P2D model uses 80 nodes in each electrode, 40 nodes in the separator domain and 100 nodes in each solid particle, which leads to a system of 16,040 equations to solve.

Figure 2 shows the voltage prediction calculated by our spectral P2D model (solid lines) vs. the Fortran FDM P2D model during a full constant current discharge at different $\mathrm{C}$ rates ranging from $1 \mathrm{C}$ to $40 \mathrm{C}$. The $\mathrm{P} 2 \mathrm{D}$ model solved using orthogonal collocation shows good agreement up to $10 \mathrm{C}$ with a $25 \mathrm{mV}(<1 \%)$ maximal error on the voltage prediction. 
Some discrepancies between the voltage predicted by the two models become significant above $20 \mathrm{C}$ with $85 \mathrm{mV}(\sim 3 \%)$ maximal voltage error at $40 \mathrm{C}$.

\begin{tabular}{lcc}
\hline Parameters & Anode & Cathode \\
\hline Thickness, $\mu m$ & 72 & 68 \\
Radius of particles, $\mu m$ & 10 & 10 \\
Solid phase volume fraction & 0.6 & 0.5 \\
Charge transfer coefficients & 0.5 & 0.5 \\
Reaction rate, $\times 10^{-11} \mathrm{~m}^{2.5} \mathrm{~mol}^{-0.5} \mathrm{~s}^{-1}$ & 5.0 & 5.0 \\
$\mathrm{Li}^{+}$diffusion coefficient, $\times 10^{-14} \mathrm{~m}^{2} . \mathrm{s}^{-1}$ & 3.9 & 10 \\
Solid-phase conductivity, $S . \mathrm{m}^{-1}$ & 100 & 10 \\
Bruggeman exponent & 1.5 & 1.5 \\
Solid-phase max concentration, mol. $\mathrm{m}^{-3}$ & 24,983 & 51,218 \\
Initial stoichiometry $x$ or $y$ & 0.6 & 0.5 \\
\hline & \multicolumn{2}{c}{ Electrolyte/Sep. } \\
\hline Separator thickness, $\mu m$ & \multicolumn{2}{c}{1} \\
Electrolyte volume fraction & \multicolumn{2}{c}{1000} \\
Initial electrolyte concentration, mol. $\mathrm{m}^{-3}$ & \multicolumn{2}{c}{$7.8 \times 10^{-10}$} \\
Electrolyte diffusion coefficient, $\mathrm{m}^{2} . \mathrm{s}^{-1}$ & \multicolumn{2}{c}{0.5} \\
Electrolyte ionic conductivity, $S . m^{-1}$ & \multicolumn{2}{c}{0.4} \\
Li ${ }^{+}$transference number in electrolyte &
\end{tabular}

Table 1: Model parameters used in the simulation.

We believe that these discrepancies at high C-rates are due to mismatches between the two models. The P2D model equations used are not exactly the same as the equations originally derived by Doyle et al. [1], which makes the comparison between the two models inexact. Another possible explanation is that the spectral P2D model does not use sufficient collocation points to converge to the same solution. However, little impact on the voltage when increasing the node density in each domain was noticed, except in solid particles at high C-rate where the concentration profile becomes very sharp near the particles' surface. This explains why more collocation points were used in the solid particles compared to the other domains.

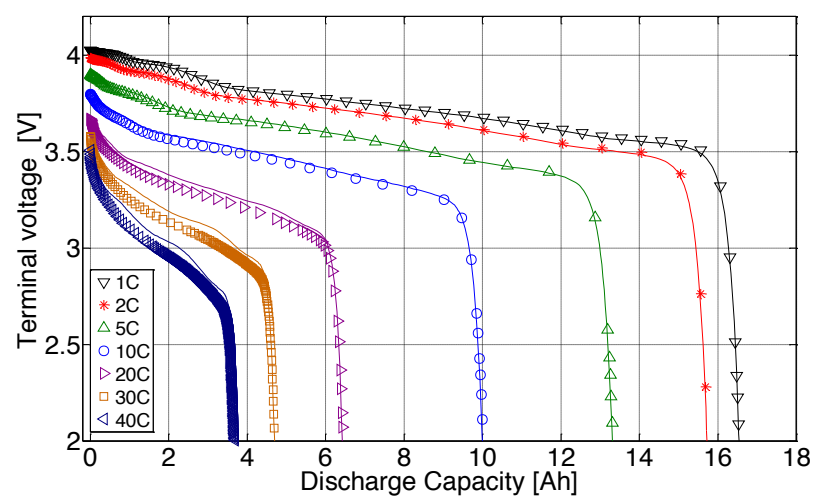

Figure 2: Battery voltage discharge curves at different $\mathrm{C}$-rates using the Fortran FDM P2D model (markers) and the Matlab Spectral P2D model (solid line).

However the main aim of this work is to reduce the computational burden, rather than achieving a direct match between models. We are confident that the spectral P2D model solved with a few collocation points will predict accurate results that may be combined with an efficient parameter estimation algorithm and used in an observer to track the voltage of commercial cells.

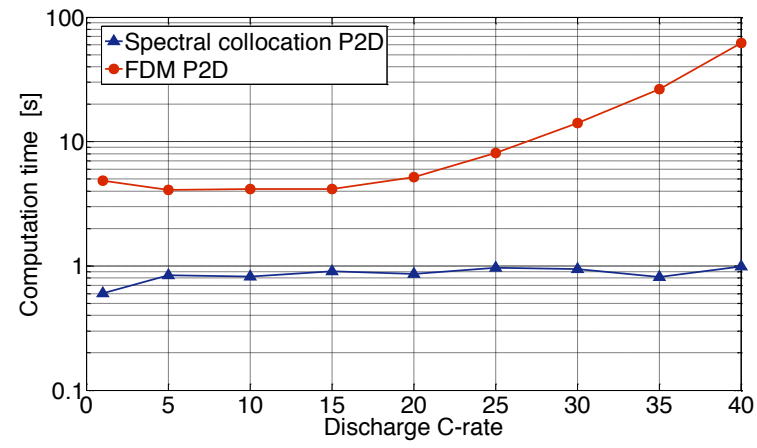

Figure 3: Computational times of a full discharge (cut-off voltage: $2 \mathrm{~V}$ ) at different $\mathrm{C}$-rates.

Figure 3 shows the computation time required by both models to solve a full constant current discharge as a function of Crate when solved on a $3.40 \mathrm{GHz}$ desktop computer. At low to medium C-rates $(<20 \mathrm{C})$, the spectral P2D model implemented in Matlab (an interpreted language) is about 4 times faster than the FDM P2D model implemented in compiled Fortran code. At higher C-rates, the computation time difference becomes even more significant with a spectral P2D model 60 times faster than the FDM P2D model at 40C. These results confirm the potential of the spectral orthogonal collocation approach for solving the P2D model for on-line estimation. It is likely that computational time of the spectral P2D model will be further reduced by providing the Jacobian of the model to the Matlab solver as is done in the Fortran code.

(a)

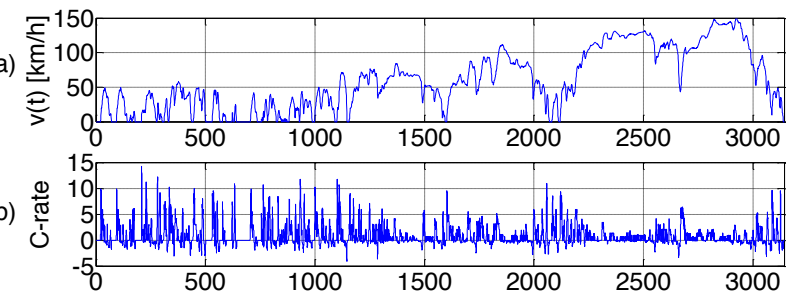

(c)

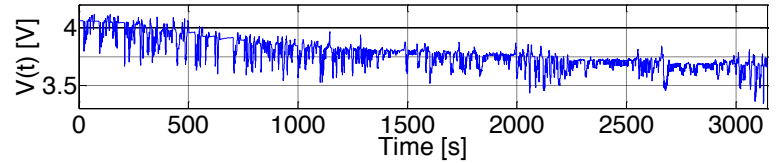

Figure 4: Voltage prediction using the Matlab spectral P2D model during a Common Artemis Driving Cycle.

Figure 4 shows the voltage predicted by the P2D model solved by the spectral orthogonal collocation method during the Common Artemis Driving Cycle. It is assumed that the current intensity is proportional to the acceleration of the vehicle and $20 \%$ of the breaking acceleration is regenerated to charge the battery. The $3143 \mathrm{~s}$ simulation was solved in $53 \mathrm{~s}$ on the computer previously mentioned, which means that a $17 \mathrm{~ms}$ computation time is required on average to solve $1 \mathrm{~s}$ of simulation. This result is encouraging because it suggests that the P2D model solved by spectral orthogonal collocation can be used for real-time state estimation. 


\section{Conclusion}

The physics-based pseudo-2D battery model was solved using the spectral orthogonal collocation method. The DAE statespace model obtained using the spectral approach is much smaller and faster to solve (by a factor of 4 to 60) compared to the one resulting from the use of the finite difference method, while maintaining accuracy up to high C-rates (40C). The spectral approach makes the real-time solution of the P2D model possible for on-line battery state estimation. Future work will focus on speeding up the model by deriving the Jacobian of the DAEs' system and providing it to the Matlab solver. The model will also be validated against experimental data and a parameter estimation algorithm will be implemented to better fit real battery behaviour. Finally, the design of an observer using the P2D model solved by orthogonal collocation method will be investigated for on-line battery state monitoring.

\section{Acknowledgements}

This work is funded by Samsung Electronics Co. Ltd. through a Global Research Outreach award. We are extremely grateful for the support of Samsung in this work.

\section{References}

[1] M. Doyle, T. F. Fuller, and J. Newman, "Modeling of Galvanostatic Charge and Discharge of the Lithium/Polymer/Insertion Cell," J. Electrochem. Soc., vol. 140, no. 6, p. 1526, (1993).

[2] K. Smith and C.-Y. Wang, "Power and thermal characterization of a lithium-ion battery pack for hybridelectric vehicles," J. Power. Sources., vol. 160, no. 1, pp. 662-673, (2006).

[3] V. R. Subramanian, J. a. Ritter, and R. E. White, "Approximate Solutions for Galvanostatic Discharge of Spherical Particles I. Constant Diffusion Coefficient," J. Electrochem. Soc., vol. 148, no. 11, p. E444, (2001).

[4] M. Guo and R. E. White, "An approximate solution for solid-phase diffusion in a spherical particle in physicsbased Li-ion cell models," J. Power. Sources., vol. 198, pp. 322-328, (2012).

[5] K. a. Smith, C. D. Rahn, and C.-Y. Wang, "Model Order Reduction of 1D Diffusion Systems Via Residue Grouping," J. Dyn. Syst. Meas. Control., vol. 130, no. 1, p. 011012, (2008).

[6] N. Chaturvedi, R. Klein, J. Christensen, J. Ahmed, and A. Kojic, "Algorithms for Advanced BatteryManagement Systems," IEEE. Contr. Syst. Mag., vol. 30, no. 3, pp. 49-68, (2010).

[7] K. A. Smith, C. D. Rahn, and C.-Y. Wang, "Control oriented 1D electrochemical model of lithium ion battery," Energy Conversion and Management, vol. 48, no. 9, pp. 2565-2578, (2007).

[8] L. Cai and R. E. White, "Reduction of Model Order Based on Proper Orthogonal Decomposition for Lithium-Ion Battery Simulations," J. Electrochem. Soc., vol. 156, no. 3, p. A154, (2009).
[9] T.-S. Dao, C. P. Vyasarayani, and J. McPhee, "Simplification and order reduction of lithium-ion battery model based on porous-electrode theory," $J$. Power. Sources., vol. 198, pp. 329-337, (2012).

[10] P. W. C. Northrop, V. Ramadesigan, S. De, and V. R. Subramanian, "Coordinate Transformation, Orthogonal Collocation, Model Reformulation and Simulation of Electrochemical-Thermal Behavior of Lithium-Ion Battery Stacks," J. Electrochem. Soc., vol. 158, no. 12, p. A1461, (2011).

[11] L. Cai and R. E. White, "Lithium ion cell modeling using orthogonal collocation on finite elements," $J$. Power. Sources., vol. 217, pp. 248-255, (2012).

[12] L. N. Trefethen, "Spectral Methods in MATLAB," (2000)

[13] J. A. C. Weideman and S. C. Reddy, “A MATLAB Differentiation Matrix Suite," ACM. Trans. Math. Softw., vol. 26, no. 4, pp. 465-519, (2000).

\section{List of Symbols}

$A \quad$ Electrode surface area $\left[\mathrm{m}^{2}\right]$

$a_{s} \quad$ Specific interfacial area $\left[\mathrm{m}^{-1}\right]$

c Local Li concentration of a phase $\left[\mathrm{mol} . \mathrm{m}^{-3}\right]$

$D$ Diffusion coefficient in a phase $\left[\mathrm{m}^{2} . \mathrm{s}^{-1}\right]$

$\mathcal{F} \quad$ Faraday's constant [96487 C. ol $^{-1}$ ]

$I_{\text {app }} \quad$ Applied current $[A]$

$i_{0} \quad$ Exchange current density $\left[A . \mathrm{m}^{-2}\right]$

$j^{L i} \quad$ Volumetric reaction rate $\left[A . m^{-3}\right]$

$k \quad$ Reaction rate constant $\left[\mathrm{m}^{2.5} \mathrm{~mol}^{-0.5} \mathrm{~s}^{-1}\right]$

$L \quad$ Thickness of the cell $[m]$

$p \quad$ Bruggeman porosity correction exponent

$R \quad$ Universal gas constant [8.314 J. $\mathrm{mol}^{-1} \mathrm{~K}^{-1}$ ]

$R_{s} \quad$ Average radius of electrodes' solid particles [m]

$r \quad$ Radial coordinates in solid particles $[\mathrm{m}]$

$T$ Temperature $[K]$

$t_{+}^{0} \quad$ Lithium ion transference number in electrolyte

$U^{o c p} \quad$ Open-circuit potential $[V]$

$x \quad$ Coordinate along the cell thickness $[\mathrm{m}]$

\section{Greek symbols}

$\alpha \quad$ Charge transfer coefficient

$\delta \quad$ Thickness of a domain $[m]$

$\epsilon \quad$ Volume fraction

$\eta \quad$ Overpotential $[V]$

$\theta \quad$ Active material stoichiometry

$\kappa \quad$ Ionic conductivity of the electrolyte $\left[S . \mathrm{m}^{-1}\right]$

$\sigma \quad$ Conductivity of the solid-phase $\left[S . \mathrm{m}^{-1}\right]$

$\phi \quad$ Local potential of a phase [V]

\section{Subscripts}

Anode

Separator

Cathode

anodic

cathodic

Electrolyte phase

Solid-phase eff

\section{Superscripts}

$\max$ maximum

surf Particles' surface 